\title{
Molecular docking analysis of curcumin analogues as human neutrophil elastase inhibitors
}

\author{
Radhakrishnan Narayanaswamy ${ }^{1}$, Lam Kok Wai ${ }^{2}$, Faridah Abas ${ }^{1,4}$ and Intan Safinar \\ Ismail1,3
}

${ }^{1}$ Laboratory of Natural Products, Institute of Bioscience (IBS), Universiti Putra Malaysia (UPM), 43400 Serdang, Selangor, Malaysia; ${ }^{2}$ Faculty of Pharmacy, Universiti Kebangsaan Malaysia (UKM), Jalan Raja Muda Abdul Aziz, 50300 Kuala Lumpur, Malaysia; ${ }^{3}$ Department of Chemistry, Faculty of Science, Universiti Putra Malaysia (UPM), 43400 Serdang, Selangor, Malaysia; ${ }^{4}$ Department of Food Science, Faculty of Food Science and Technology, Universiti Putra Malaysia (UPM), 43400 Serdang, Selangor, Malaysia.

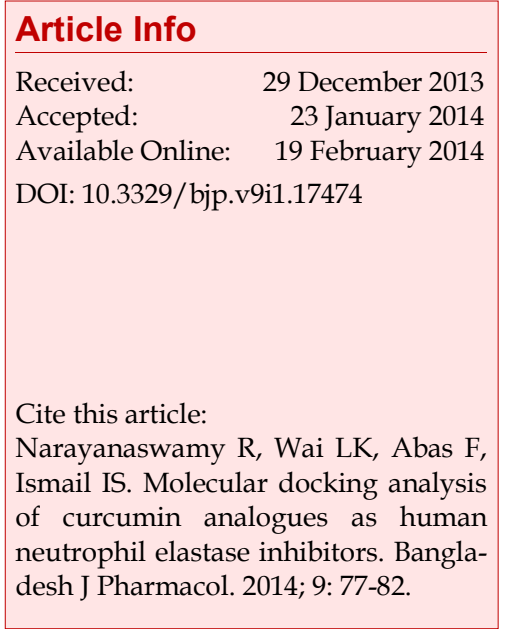

\begin{abstract}
In the present study, we aimed to dock 17 different ligands of curcumin analogues with that of human neutrophil elastase. Molecular descriptors analysis using Molinspiration online tool was carried out including investigation on human neutrophil elastase putative binding sites using Discovery Studio. The molecular physicochemical analysis revealed that all of the curcumin analogues complied well with the five rules of thumb. With regard to bioactivity score, compound $\mathbf{1 7}$ has exhibited least score towards nuclear receptor ligand (0.05) and enzyme inhibitor (0.10) compared to all other ligands. Compounds 2, 4 and 13 exhibited the maximum interaction energy $(-40 \mathrm{kcal} / \mathrm{mol})$. Interestingly, seven compounds namely 3, 11-14, 16 and 17 interacted well with Arg147 amino acid residue. The present study outcomes therefore might provide new insight in understanding these 17 curcumin analogues as potential candidates for human neutrophil elastase inhibitory agents.
\end{abstract}

\section{Introduction}

Curcumin has a salient chemical structure containing two ferulic acid residues joined by a methylene bridge. It has two hydrophobic phenyl domains that are connected by a flexible (seven carbon) linker. Although curcumin has unique structure feature, however it lacks in stability (Wang et al., 1997) and bioavailability features (Gupta et al., 2011). Hence, numerous curcumin analogues were synthesized wherein to-date several natural and synthetic analogues of curcumin have been reported to possess therapeutic applications (Furness et al., 2005) in which some of them are used as potential anti-inflammatory agents (Mukhopadhyay et al., 1982). Molecular docking studies have found that curcumin can adopt many different structural conformations suitable for maximizing hydrophobic contacts with the macromolecule to which it is bound. For instance, the phenyl rings of curcumin can participate in 0-0 van der Waals interactions with aromatic amino acid side chains of macromolecules. Even though curcumin generally is hydrophobic in nature, the phenolic and carbonyl moiety located at the end and the center of the molecule could participate in hydrogen bonding with that of targeted macromolecules. The keto-enol tautomerization also contributes to curcumin additional unique chemical functionality with a strong and direct electrostatic interaction to increase favorable free energies of association.

Molecular modeling study carried out by Nirmal et al. (2008) showed that curcumin could bind at the active site of bovine pancreatic phospholipase A2 (PLA2) which is one of the key enzymes in inflammatory 
pathway. A few other reports also supported that curcumin and its analogues could bind to various enzymes which includes human immunodeficiency virus type-I protease (Sui et al., 1993), cyclooxygenase-1 (Selvam et al., 2005), DNA polymerase $\lambda$ (Takeuchi et al., 2006), platelet-12-lipoxygenase (Jankun et al., 2006), cyclooxygenase-2 (Padhye et al., 2009), DNA methyl transferase-1 (Liu et al., 2009), xanthine oxidase (Shen and Ji, 2009), dipeptydyl peptidase-4 (Istyastono, 2009), glycogen synthase kinase-3 $\beta$ (Bustanji et al., 2009), ribonuclease A (Sahoo et al., 2009), glyoxalase-I (Liu et al., 2010), protein kinase C (Majhi et al., 2010) and matrix metalloproteinases (Girija et al., 2010).

Although curcumin and analogues have been reported to bind with various enzymes, till date no report is available for human neutrophil elastase inhibitory activity. Human neutrophil elastase has recently gained a lot of attention worldwide as it has a potential therapeutic target for the treatment of inflammation related diseases. This prompted us to carry out the present study on a selected 17 curcumin analogues among the 47 which were grouped under three major types of a) pentadiene-3-one, b) dibenzyldiene cyclohexanone and c) dibenzyldiene cyclopentanone. Some of these compounds were subsequently reported for various biological activities such as anti-inflammatory, anti-oxidant, anti-tyrosinase (Lee et al., 2009), chemotactic (Jantan et al., 2012) and anti-melanogenesis (Hosoya et al., 2012).

The selected compounds are a) 2,5 -bis (2,3-dimethoxy benzylidene) cyclopentanone; b) 2,6 -bis (3,4,5-trimethoxybenzylidene) cyclohexanone; c) 2,6-bis (2,4,6-trimetho -xybenzylidene) cyclohexanone; d) 2,6-bis (2,3,4-trimethoxybenzylidene) cyclohexanone; e) 2,6-bis (2,6-dimethoxybenzylidene) cyclohexanone; f) 2,6-bis (2,5-dimethoxybenzylidene) cyclohexanone; g) 2,6-bis (2,4-dimethoxybenzylidene) cyclohexanone; h) 2,6-bis (2,3-dimethoxybenzylidene) cyclohexanone; i) 2,6-bis (benzylidene) cyclohexanone; j) 2,6-bis (4-hydroxy benzylidene) cyclohexanone; k) 2,6-bis (4-hydroxy-3-methoxy benzylidene) cyclohexanone; 1) 1,5-diphenyl-(E, E)-1,4 pentadiene-3-one; 1) 1,5-bis (2,4,6-trimethoxyphenyl)-1,4 -pentadiene-3-one; m) 1,5-bis (2,6-dimethoxyphenyl)-1,4 -pentadiene-3-one; n) 1,5-bis (2,4-dimethoxyphenyl)-1,4 -pentadiene-3-one; o) 1,5-bis (2,3-dimethoxyphenyl)-1,4pentadiene-3-one and p) 1,5-bis (2-hydroxyphenyl)-1,4pentadiene-3-one, were evaluated on the docking behaviour of human neutronphil elastase. Investigation was also done on human neutrophil elastase putative binding sites using Discovery Studio Version 3.1 whereby the results from the present study would give some useful information for the researchers to design potent and selective human neutrophil elastase inhibitors of curcumin analogues in the near future.

\section{Materials and Methods}

Ligand preparation
Chemical structures of ligands namely a) 2,5-bis (2,3dimethoxy benzylidene) cyclopentanone [Chemspider ID 1450996]; b) 2,6-bis (3,4,5-trimethoxy benzylidene) cyclohexanone [Chemspider ID 1372795]; c) 2,6-bis (2,4,6-trimethoxy benzylidene) cyclohexanone [Chemspider ID 24669292]; d) 2,6-bis (2,3,4-trimethoxy benzylidene) cyclohexanone

[Chemspider ID 4480275]; e) 2,6-bis (2,6-dimethoxy benzylidene) cyclohexanone [Chemspider ID 3373346]; f) 2,6-bis (2,5-dimethoxy benzylidene) cyclohexanone [Chemspider ID 1415781]; g) 2,6-bis (2,4-dimethoxy benzylidene) cyclohexanone [Chemspider ID 1500590]; h) 2,6-bis (2,3-dimethoxy benzylidene) cyclohexanone [Chemspider ID 2530853]; i) 2,6-bis (benzylidene) cyclohexanone [Chemspider ID 1266977]; j) 2,6-bis (4hydroxy benzylidene) cyclohexanone [Chemspider ID 1468601]; k) 2,6-bis (4-hydroxy-3-methoxy benzylidene) cyclohexanone [Chemspider ID 1266900] and 1) 1,5diphenyl-( $E, E)-1,4$ pentadiene-3-one [Chemspider ID 555548] were retrieved from Chemspider compound database (www.chemspider.com). Unavailable three dimensional structures of $\mathrm{m}$ ) 1,5-bis (2,4,6-trimethoxy phenyl)-1,4-pentadiene-3-one; n) 1,5-bis (2,6-dimethoxy phenyl)-1,4-pentadiene-3-one; o) 1,5-bis (2,4-dimethoxy phenyl)-1,4-pentadiene-3-one; p) 1,5-bis (2,3-dimethoxy phenyl)-1,4-pentadiene-3-one and q) 1,5-bis (2-hydroxy phenyl)-1,4-pentadiene-3-one were generated using ACD (Anonymous., 2009).

\section{Target protein identification and preparation}

The three dimensional structure of the human neutrophil elastase (PDB ID: 1H1B with resolution of $2.00 \AA$ ) was obtained from the Research collaborator for structural bioinformatics (RCSB) Protein data bank (www.rcsb.org). A chain of protein was pre-processed separately by deleting the chain B, ligand, as well as the crystallographically observed water molecules (water without hydrogen bonds).

\section{Molecular descriptors calculation}

Molinspiration online database was used to calculate thirteen descriptors (www.molinspiration.com), which are $\log \mathrm{P}$, polar surface area, molecular weight, number of atoms, number of $\mathrm{O}$ or $\mathrm{N}$, number of $\mathrm{OH}$ or $\mathrm{NH}$, number of rotatable bonds, volume, drug-likeness includes G protein coupled receptors (GPCR) ligand, ion channel modulator, kinase inhibitor and nuclear receptor ligand, and number of violations to Lipinski's rule, for all selected ligands except two (2,6-bis (4-hydro -xybenzylidene) cyclohexanone and 2,6-bis (4-hydroxy3-methoxy benzylidene) cyclohexanone) which have been reported earlier.

\section{Docking studies}

Docking studies were carried out on the crystal structure of human neutrophil elastase retrieved from Protein Data Bank using the CDOCKER protocol under the protein-ligand interaction section in Discovery Studio ${ }^{\circledR} 3.1$ (Accelrys, San Diego, USA). In general, 
CDOCKER is a grid-based molecular docking method that employs CHARMM force fields. This protein was firstly held rigid while the ligands were $\mathbf{7 8}$ Bangladesh J Pharmacol 2014; 9: 77-82 allowed to flex during the refinement. Two hundred random ligand conformations were then generated from the initial ligand structure through high temperature molecular dynamics, followed by random rotations, refinement by grid-based (GRID I) simulated annealing, and a final grid-based or full force field minimisation ( $\mathrm{Wu}$ et al., 2003). In this experiment, the ligand was heated to a temperature of $700 \mathrm{~K}$ in 2,000 steps. The cooling steps were set to 5,000 steps with $300 \mathrm{~K}$ cooling temperature. The grid extension was set to $10 \AA$.

Hydrogen atoms were added to the structure and all ionisable residues were set at their default protonation state at a neutral $\mathrm{pH}$. For each ligand, top ten ligand binding poses were ranked according to their CDOCKER energies, and the predicted binding interactions were analyzed.

\section{Results and Discussion}

Medicinal and computational chemists proposed the concept of "drug-likeness", which is valuable tool to select more promising lead candidates by predicting (or) evaluating their drug-likeness property in the early stage of drug iscovery and development (Lipinski et al., 2001). The rules of drug-likeness were proposed by analyzing the physico-chemical properties of known drugs. The most famous drug-likeness filter is Lipinski's "rule-of-five". In the present study violation of Lipinski's "rule-of-five" was recorded using Molinspiration online tool, if $\log >5$, Molecular eight $(\mathrm{MW})>500$, number of $\mathrm{N}$ and $\mathrm{O}$ (hydrogen bond acceptors) $>10$, number of $\mathrm{OH}$ and $\mathrm{NH}$ (hydrogen bond donors) $>5$ and number of rotatable bonds $>15$. Interestingly, all the 15 ligands complied with five rules of thumb as tabulated in Table I.

The molecular physicochemical and the drug-likeness properties of two ligands which are 2, 6-bis (4-hydroxy benzylidene) cyclohexanone (Lam et al., 2012) and 2, 6bis (4-hydroxy-3-methoxy benzylidene) cyclohexanone (Lam et al., 2012; Sangeetha et al., 2013) were already been reported. In addition, Sangeetha et al. (2013) also has reported the molecular physicochemical and the drug-likeness properties of curcumin, the parent compound.

With regard to bioactivity score, 1, 5-bis (2-hydroxy phenyl)-1, 4-pentadiene-3-one (compound 17) has exhibited least score towards nuclear receptor ligand $(0.05)$ and enzyme inhibitor (0.10) compared to all other ligands as shown in the Table II.

Human neutrophil elastase is a $30 \mathrm{kD}$ molecular weight glycoprotein and synthesized as zymogen (pro-form), which becomes active form after post-translation modification (Pham, 2006). Human neutrophil elastase has specificity towards small hydrophobic amino acids. The potent catalytic activity is facilitated by a catalytic triad that is conserved among all serine proteinase, which consists of His, Asp and Ser amino acid residues forming a charge relay system. During proteolysis, the side chain of the peptide is located in the S1 specificity pocket while its backbone carbonyl is placed in the 'oxy anion hole' and forms hydrogen bonds with the amino group of Gly193 and Ser195 amino acid residues, thus stabilizing the charge transition state (Bode et al., 1989).

Several studies have been conducted recently to examine the binding interaction of inhibitors to the human neutrophil elastase structure (Siedle et al., 2002; Sivamani et al., 2012; Lucas et al., 2013; Crocetti et al., 2013; Radhakrishnan et al., 2013). In the present study, the aim is to understand the binding interactions between curcumin analogues and the active site of the human neutrophil elastase. The crystal structure of human neutrophil elastase $(1 \mathrm{H} 1 \mathrm{~B}$ with resolution of $2.00 \mathrm{~A}) \square$ was retrieved and then prepared according to the standard protocol implemented in Discovery Studio ${ }^{\circledR}$ 3.1. Subsequently the docking and interaction results were tabulated in Table III, in which 2,6-bis (3,4,5-trimethoxy benzylidene) cyclohexanone (compound 2), 2,6-bis (2,3,4-trimethoxy benzylidene) cyclohexanone (compound 4) and 1,5-bis (2,4,6-trimethoxy phenyl)-1,4-pentadiene-3-one (compound 13) exhibited the maximum interaction energy $(-40 \mathrm{kcal} /$ mol). In contrast, 1, 5-diphenyl-(E, E)-1, 4 pentadiene-3one (compound 12) and 2, 6-bis (4-hydroxy-3-methoxy benzylidene) cyclohexanone (compound 9) showed very least interaction energy of -23.7 and $-24.7 \mathrm{kcal} / \mathrm{mol}$ respectively compared to all other ligands ( -28.3 to -37.3 $\mathrm{kcal} / \mathrm{mol}$ ) as shown in the Table III.

Whereby 1) 2,5-bis (2,3-dimethoxy benzylidene) cyclopentanone, 2) 2,6-bis (3,4,5-trimethoxy benzylidene) cyclohexanone, 3) 2,6-bis (2,4,6-trimethoxy benzylidene) cyclohexanone, 4) 2,6-bis (2,3,4-trimethoxy benzylidene) cyclohexanone, 5) 2,6-bis (2,6-dimethoxy benzylidene) cyclohexanone, 6) 2,6-bis (2,5-dimethoxy benzylidene) cyclohexanone, 7) 2,6-bis (2,4-dimethoxy benzylidene) cyclohexanone and 8) 2,6-bis (2,3-dimethoxy benzylidene) cyclohexanone. Hydrogen atoms have been omitted in the two dimensional diagram for better clarity. The pink line indicates the hydrogen bond interaction. In addition to these, bond distances are indicated in angstroms (A) $\square$ unit.

The ligands are 9) 2,6-bis (benzylidene) cyclohexanone, 10) 2,6-bis (4-hydroxy benzylidene) cyclohexanone, 11) 2,6-bis (4-hydroxy-3-methoxy benzylidene) cyclohexanone, 12) 1,5-diphenyl-(E, $E)-1,4$ pentadiene-3 -one, 13) 1,5-bis (2,4,6-trimethoxy phenyl)-1,4-penta-diene-3one, 14) 1,5-bis (2,6-dimethoxy phenyl)-1,4-pentadiene3-one, 15) 1,5-bis (2,4-dimethoxy phenyl)-1,4-pentadiene-3-one and 16) 1,5-bis (2,3-dimethoxy phenyl)-1,4- 


\section{Table I}

Molecular descriptors analysis of 15 ligands using Molinspiration online software tool

\begin{tabular}{|c|c|c|c|c|c|c|c|c|c|}
\hline Ligand & $\log A a$ & TPSAb & Natomsc & MWd & noNe & nOH NHf & Nviolationsg & Nrotbh & Volumei \\
\hline Compound 1 & 3.74 & 54.00 & 28 & 380 & 5 & 0 & 0 & 6 & 354.2 \\
\hline Compound 2 & 4.23 & 72.47 & 33 & 454 & 7 & 0 & 0 & 8 & 422.1 \\
\hline Compound 3 & 4.66 & 72.47 & 33 & 454 & 7 & 0 & 0 & 8 & 422.1 \\
\hline Compound 4 & 4.26 & 72.47 & 33 & 454 & 7 & 0 & 0 & 8 & 422.1 \\
\hline Compound 5 & 4.64 & 54.00 & 29 & 394 & 5 & 0 & 0 & 6 & 371.0 \\
\hline Compound 6 & 4.69 & 54.00 & 29 & 394 & 5 & 0 & 0 & 6 & 371.0 \\
\hline Compound 7 & 4.69 & 54.00 & 29 & 394 & 5 & 0 & 0 & 6 & 371.0 \\
\hline Compound 8 & 4.24 & 54.00 & 29 & 394 & 5 & 0 & 0 & 6 & 371.0 \\
\hline Compound 9 & 4.96 & 17.00 & 21 & 274 & 1 & 0 & 0 & 2 & 268.8 \\
\hline Compound 12 & 4.18 & 17.07 & 18 & 234 & 1 & 0 & 0 & 4 & 229.2 \\
\hline Compound 13 & 3.87 & 72.47 & 30 & 414 & 7 & 0 & 0 & 10 & 382.5 \\
\hline Compound 14 & 3.86 & 54.00 & 26 & 354 & 5 & 0 & 0 & 8 & 331.4 \\
\hline Compound 15 & 3.90 & 54.00 & 26 & 354 & 5 & 0 & 0 & 8 & 331.4 \\
\hline Compound 16 & 3.46 & 54.00 & 26 & 354 & 5 & 0 & 0 & 8 & 331.4 \\
\hline Compound 17 & 3.70 & 57.00 & 20 & 266 & 3 & 2 & 0 & 4 & 245.3 \\
\hline
\end{tabular}

\section{Table II}

\section{Bioactivity score calculation of 15 ligands using Molinspiration online software tool}

\begin{tabular}{|c|c|c|c|c|c|c|}
\hline Ligand & $\begin{array}{l}\text { GPCR lig- } \\
\text { and }\end{array}$ & $\begin{array}{l}\text { Ion channel } \\
\text { modulator }\end{array}$ & $\begin{array}{l}\text { Kinase in- } \\
\text { hibitor }\end{array}$ & $\begin{array}{c}\text { Nuclear receptor } \\
\text { ligand }\end{array}$ & $\begin{array}{l}\text { Protease inhib- } \\
\text { itor }\end{array}$ & $\begin{array}{l}\text { Enzyme inhibi- } \\
\text { tor }\end{array}$ \\
\hline Compound 1 & -0.29 & -0.25 & -0.38 & -0.11 & -0.13 & -0.07 \\
\hline Compound 2 & -0.12 & -0.24 & -0.28 & -0.13 & -0.08 & -0.02 \\
\hline Compound 3 & -0.10 & -0.27 & -0.28 & -0.08 & -0.04 & 0.01 \\
\hline Compound 4 & -0.13 & -0.27 & -0.31 & -0.16 & -0.11 & -0.06 \\
\hline Compound 5 & -0.11 & -0.29 & -0.32 & -0.11 & -0.03 & 0.01 \\
\hline Compound 6 & -0.12 & -0.29 & -0.35 & -0.06 & -0.08 & -0.05 \\
\hline Compound 7 & -0.12 & -0.29 & -0.34 & -0.05 & -0.08 & -0.04 \\
\hline Compound 8 & -0.13 & -0.29 & -0.36 & -0.14 & -0.10 & -0.06 \\
\hline Compound 9 & -0.14 & -0.26 & -0.44 & -0.13 & -0.09 & 0.03 \\
\hline Compound 12 & -0.25 & -0.27 & -0.37 & -0.17 & -0.27 & 0.04 \\
\hline Compound 13 & -0.08 & -0.28 & -0.14 & -0.00 & -0.04 & 0.04 \\
\hline Compound 14 & -0.08 & -0.30 & -0.16 & -0.03 & -0.04 & 0.05 \\
\hline Compound 15 & -0.09 & -0.30 & -0.18 & 0.04 & -0.09 & -0.01 \\
\hline Compound 16 & -0.11 & -0.30 & -0.20 & -0.06 & -0.11 & -0.03 \\
\hline Compound 17 & -0.13 & -0.26 & -0.23 & 0.05 & -0.15 & 0.10 \\
\hline
\end{tabular}

pentadiene-3-one. Hydrogen atoms have been omitted in the two dimensional diagram for better clarity. The pink line indicates charge interaction. In addition to these, bond distances are indicated in angstroms (A) $\square$ unit. The 17 ligand is 1, 5 -bis (2-hydroxy phenyl)-
1, 4-pentadiene-3-one. Hydrogen atoms have been omitted in the two dimensional diagram for better clarity. The pink line indicates the charge interaction. In addition to these, bond distances are indicated in angstroms (A) $\square$ unit. 
Table III

The interaction energy analysis of 17 ligands docked with that of human neutrophil elastase using Discovery Studio® 3.1

\begin{tabular}{|c|c|c|c|}
\hline Ligand name & $\begin{array}{l}\text { cDocker interaction energy }{ }^{a} \\
(\mathrm{kcal} / \mathrm{mol})\end{array}$ & $\begin{array}{l}\text { Interaction with amino acid } \\
\text { residue }\end{array}$ & Bond distance $(\mathrm{A} \square)$ \\
\hline Compound 1 & -29.9 & No interactions & - \\
\hline Compound 2 & -40.0 & Phe192 & 3.2 \\
\hline Compound 3 & -37.3 & Arg147 & 2.8 \\
\hline Compound 4 & -40.0 & Phe192 & 3.0 \\
\hline Compound 5 & -33.5 & Gly219 & 3.0 \\
\hline Compound 6 & -35.1 & Val216 & 3.0 \\
\hline Compound 7 & -37.7 & No interactions & - \\
\hline Compound 8 & -32.4 & No interactions & - \\
\hline Compound 9 & -24.7 & No interactions & - \\
\hline Compound 10 & -30.2 & No interactions & - \\
\hline Compound 11 & -33.8 & Arg177, Phe192 & $3.2,3.1$ \\
\hline Compound 12 & -23.7 & Arg147 & 2.7 \\
\hline Compound 13 & -40.1 & Arg147 & Not analyzed \\
\hline Compound 14 & -33.7 & Arg147, Gly219 & $2.9,3.0$ \\
\hline Compound 15 & -36.8 & No interactions & - \\
\hline Compound 16 & -35.1 & Arg147, Gly218 & $2.8,3.0$ \\
\hline Compound 17 & -28.3 & Arg147, Val216 & $2.8,1.9$ \\
\hline
\end{tabular}

Among the 17 ligands studied, five compounds namely compounds 7-10 (2,6-bis (2,4-dimethoxy benzylidene) cyclohexanone; 2,6-bis (2,3-dimethoxy benzylidene) cyclohexanone; 2,6-bis (benzylidene) cyclohexanone; 2,6 -bis (4-hydroxy benzylidene) cyclohexanone) and 15 (1,5-bis (2,4-dimethoxy phenyl)-1,4-pentadiene-3-one) did not exhibit any interaction with any of amino acid residues active site (Table III). On other hand, we found that seven ligands $(3,11-14,16,17)$ interacted with Arg147 amino acid residue. Interestingly, none of the ligands was found to be interacted with Ser195 amino acid residue. No reports are available for elastase inhibitory activity of these 17 curcumin analogues till date. However parent compound (curcumin) has been reported to inhibit metalloelastase (MMP-12) using both, molecular docking and wet laboratory studies by Singh et al. (2012).

\section{Conclusion}

We strongly believe that the results of this present study might provide new insight in understanding these 17 ligands (curcumin analogues) as potential candidates for human neutrophil elastase inhibitory agents. To our knowledge, we are the first to report the binding of these 12 curcumin analogues with that of human neutrophil elastase structure even though various enzymes were known to bind to the parent compound (curcumin).

\section{Acknowledgement}

The first author (RN) would like to thank the Research Management Center (RMC) of Universiti Putra Malaysia (UPM) for the Post-Doctoral financial support.

\section{References}

Anonymous, Molinspiration Database ( $\mathrm{ht} \mathrm{t} \mathrm{p}: / /$ www.molinspiration.com).

Anonymous. ACD, ChemSketch Version 12, Advanced Chemistry Development Inc., Toronto, ON, Canada, 2009 (http://www.acdlabs.com/download/chemsk.html).

Anonymous. Chemspider ( http://www.chemspider.com).

Anonymous. Research collaborator for structural bioinformatics Protein data bank (RCSB PDB) (http:// www.rcsb.org).

Bode W, Meyer EJr, Powers JC. Human leukocyte and porcine pancreatic elastase: X-ray crystal structures, mechanism, substrate specificity, and mechanism-based inhibitors. Biochemistry 1989; 28: 1951-63.

Bustanji Y, Taha MO, Almasri IM, Al-Ghussein MA, Mohammad MK, Alkhatib HS. Inhibition of glycogen synthase kinase by curcumin: Investigation by simulated molecular docking and subsequent in vitro/in vivo evaluation. J Enzyme Inhib Med Chem. 2009; 24: 771-78.

Crocetti L, Schepetkin IA, Cilibrizzi A, Graziano A, Vergelli C, Giomi D, Khlebnikov AI, Quinn MT, Giovannoni MP. Optimization of N-benzoylindazole derivatives as inhibitors of human neutrophil elastase. J Med Chem. 2013; 56: 6259-72. 
Furness MS, Robinson TP, Ehlers T, Hubbard RB, Arbiser JL, G o ld s m i th D J, B o w e n J P. A n t i a n gi o g enic agents: Studies on fumagillin and curcumin analogs. Curr Pharm Des. 2005 ; 11: 357-73.

Girija CR, Karunakar P, Poojari CS, Begum NS, Syed AA. Molecular docking studies of curcumin derivatives with multiple protein targets for procarcinogen activating enzyme inhibition. J Proteomics Bioinform. 2010; 3: 200-03.

Gupta SC, Prasad S, Kim JH, Patchva S, Webb LJ, Priyadarsini IK, Aggarwal BB. Multi-targeting by curcumin as revealed by molecular interaction studies. Nat Prod Rep. 2011; 28: 1937-55.

Hosoya T, Nakata A, Yamasaki F, Abas F, Shaari K, Lajis NH, Morita H. Curcumin-like diarylpentanoid analogues as melanogenesis inhibitors. J Nat Med. 2012; 66: 166-76.

Istyastono EP. Docking studies of curcumin as a potential lead compound to develop novel dipeptydyl peptidase-4 inhibitors. Indo J Chem. 2009; 9: 132-36.

Jankun J, Aleem AM, Malgorzewicz S, Szkudlarek M, Zavodszky MI, Dewitt DL, Feig M, Selman SH, SkrzypczakJankun E. Synthetic curcuminoids modulate the arachidonic acid metabolism of human platelet 12-lipoxygenase and reduce sprout formation of human endothelial cells. Mol Cancer Ther. 2006; 5: 1371-82.

Jantan I, Bukhari SN, Lajis NH, Abas F, Wai LK, Jasamai M. Effects of diarylpentanoid analogues of curcumin on chemiluminescence and chemotactic activities of phagocytes. J Pharm Pharmacol. 2012; 64: 404-12.

Lam KW, Tham CL, Liew CY, Israf DA, Syahida A, Mohd Abdul Rahman B, Lajis NH. Synthesis and evaluation of DPPH and anti-inflammatory activities of 2, 6 bis benzylidenecyclohexanone and pyrazoline derivatives. Med Chem Res. 2012; 21: 333-44.

Lee KH, Ab Aziz FH, Syahida A, Abas F, Shaari K, Israf DA, Lajis NH. Synthesis and biological evaluation of curcuminlike diarylpentanoid analogues for anti-inflammatory, antioxidant and anti-tyrosinase activities. Eur J Med Chem. 2009; 44: 3195-3200.

Lipinski CA, Lombardo F, Dominy BW, Feeney PJ. Experimental and computational approaches to estimate solubility and permeability in drug discovery and development settings. Adv Drug Deliv Rev. 2001; 46: 3-26.

Liu M, Yuan M, Luo M, Bu X, Luo HB, Hu X. Binding of curcumin with glyoxalase I: Molecular docking, molecular dynamics simulations, and kinetics analysis. Biophys Chem. 2010; 147: 28-34.

Liu Z, Xie Z, Jones W, Pavlovicz RE, Liu S, Yu J, Li PK, Lin J, Fuchs JR, Marcucci G, Li C, Chan KK. Curcumin is a potent DNA hypomethylation agent. Bioorg Med Chem Lett. 2009; 19: 706-09.

Lucas SD, Costa E, Guedes RC, Moreira R. Structure based virtual screening for discovery of novel human neutrophil elastase inhibitors. Med Res Rev. 2013; 33: E73-101.

Majhi A, Rahman GM, Panchal S, Das J. Binding of curcumin and its long chain derivatives to the activator binding domain of novel protein kinase C. Bioorg Med Chem. 2010; 18: 1591-98.
Mukhopadhyay A, Basu N, Ghatak N, Gujral PK. Antiinflammatory and irritant activities of curcumin analo-gues in rats. Agents Actions. 1982; 12: 508-15.

Nirmal N, Praba GO, Velmurugan D. Modeling studies on phospholipase A2-inhibitor complexes. Indian J Biochem Biophys. 2008; 45: 256-62.

Padhye S, Banerjee S, Chavan D, Pandye S, Swamy KV, Ali S, Li J, Dou QP, Sarkar FH. Fluorocurcumins as cyclooxygenase-2 inhibitor: Molecular docking, pharmacokinetics and tissue distribution in mice. Pharm Res. 2009; 26: 2438-45.

Pham CT. Neutrophil serine proteases: Specific regulators of inflammation. Nat Rev Immunol. 2006; 6: 541-50.

Radhakrishnan N, Lam KW, Intan SI. Molecular docking analysis of natural compounds as human neutrophil elastase (HNE) inhibitors. J Chem Pharm Res. 2013; 5: 337-41.

Sahoo BK, Gosh KS, Dasgupta S. An investigation of the molecular interactions of diacetylcurcumin with ribonuclease A. Protein Pept Lett. 2009; 16: 1485-95.

Sangeetha S, Ranjitha S, Murugan K, Ramesh kumar G. Breast cancer specific histone deacetylase inhibitors and lead discovery using molecular docking and descriptor study. Trends Bioinform. 2013; 6: 25-44.

Selvam C, Jachak SM, Thilagavathi R, Chakraborti AK. Design, synthesis, biological evaluation and molecular docking of curcumin analogues as anti-oxidant, cyclooxygenase inhibitory and anti-inflammatory agents. Bioorg Med Chem Lett. 2005; 15: 1793-97.

Shen L, Ji HF. Insights into the inhibition of xanthine oxidase by curcumin. Bioorg Med Chem Lett. 2009; 19: 5990-93.

Siedle B, Cisielski S, Murillo R, Loser B, Castro V, Klaas CA, Hucke O, Labahn A, Melzig MF, Merfort I. Sesquiterpene lactones as inhibitors of human neutrophil elastase. Bioorg Med Chem. 2002; 10: 2855-61.

Singh A, Mishra A, Gautam MK, Verma S, Goel RK. Regulation of wound strength by Curcuma longa: In silico and in vivo evidences. J Pharm Res. 2012; 5: 4734-38.

Sivamani P, Singaravelu G, Thiagarajan V, Jayalakshmi T, Rameshkumar G. Comparative molecular docking analysis of essential oil constituents as elastase inhibitors. Bioinform. 2012; 8: 457-60.

Sui Z, Salto R, Li J, Craik C, Ortiz de Montellano PR. Inhibition of the HIV-1 and HIV-2 proteases by curcumin and curmumin boron complexes. Bioorg Med Chem. 1993; 1: 415-22.

Takeuchi T, Ishidoh T, Iijima H, Kuriyama I, Shimazaki N, Koiwai O, Kuramochi K, Kobayashi S, Sugawara F, Sakaguchi K, Yoshida H, Mizushina Y. Structural relationship of curcumin derivatives binding to the BRCT domain of human DNA polymerase lambda. Genes Cells. 2006; 11: 22335.

Wang YJ, Pan MH, Cheng AL, Lin LI, Ho YS, Hsieh CY, Lin JK. Stability of curcumin in buffer solutions and characterization of its degradation products. J Pharm Biomed Anal. 1997; 15: 1867-76.

Wu G, Robertson DH, Brooks CL, Vieth M. Detailed analysis of grid-based molecular docking: A case study of Cdocker-A CHARMm-based MD docking algorithm. J Comput Chem. 2003; 24: 1549-62. 